\title{
Instability of the proximal radioulnar joint in Monteggia fractures-an experimental study
}

\author{
Achim Biewener ${ }^{1}$, Fabian Bischoff ${ }^{1}$, Tobias Rischke ${ }^{1}$, Eric Tille ${ }^{1}$, Ute Nimtschke ${ }^{2}$, Philip Kasten ${ }^{3}$, \\ Klaus-Dieter Schaser ${ }^{1,4}$ and Jörg Nowotny ${ }^{1,4^{*}}$ (I)
}

\begin{abstract}
Background: A Monteggia fracture is defined as a fracture of the proximal ulna combined with a luxation of the radial head. The aim of the present work is to evaluate the extent of instability of the radius head in the proximal radioulnar joint (PRUJ) as a function of the severity of elbow fracture and ligamentous injury in an experimental biomechanical approach.

Methods: Eight fresh-frozen cadaver arms were used. All soft tissues were removed except for the ligamentous structures of the PRUJ and forearm. A tensile force of $40 \mathrm{~N}$ was exerted laterally, anteriorly or posteriorly onto the proximal radius. The dislocation in the PRUJ was photometrically recorded and measured by two independent examiners. After manual dissection of the ligamentous structures up to the interosseous membrane, the instability was documented and subsequently measured. The following dissection levels were differentiated: intact ligamentous structures, dissection of annular ligament, oblique cord and proximal third of interosseous membrane.

Results: An anterior instability remains relatively constant until the proximal third of the interosseous membrane is dissected. The radial head already dislocates relevantly in the posterior direction after dissection of the annular ligament with an additional considerable stability anteriorly and laterally. Subsequently, the posterior instability increases less pronouncedly in regard of distal resected structures. The lateral instability increases constantly during the progressing resection of the ligamentous structures.
\end{abstract}

Conclusion: On the one hand, a complete healing of the ligament injury after functional treatment is hardly conceivable with ligamentary damage up to the level of the proximal interosseous membrane. A remaining instability of the proximal radius could therefore be a possible cause for the unsatisfactory clinical results after certain Monteggia fractures. On the other hand, the present study may give a possible explanation (i.e. early dorsal radius head dislocation after dissection of annular ligament) why the Bado II injury is the most frequent type of Monteggia fractures.

Keywords: Elbow, Monteggia fracture, Proximal radioulnar joint, Radial head dislocation, Biomechanical study, Annular ligament, Interosseous membrane, Ligament reconstruction

\section{Introduction}

A Monteggia fracture is defined as a fracture of the proximal ulna combined with a dislocation of the radial head [1]. The current operative treatment of these injuries leads to favourable clinical results in the majority of cases. However, there are some injuries that do not have a favourable

\footnotetext{
* Correspondence: joerg.nowotny@uniklinikum-dresden.de

'Orthopaedic-Traumatology Centre (OUC), Carl Gustav Carus University, Technical University Dresden, Fetscherstraße 74, 01307 Dresden, Germany

${ }^{4}$ Centre for Translational Bone, Joint and Soft Tissue Research, Technical University Dresden, Dresden, Germany

Full list of author information is available at the end of the article
}

outcome. The knowledge of the fracture morphology and its involved structures are therefore important preconditions for a successful therapy [2].

Monteggia fractures are usually associated with a dislocation in the proximal radioulnar joint (PRUJ) $[2,3]$. The most commonly used classification according to Bado describes in four subtypes the direction of the radius head dislocation and thus the angulation of the ulna fracture [4]. The posterior Monteggia injury (Bado type II) is additionally classified according to Jupiter into four subtypes and describes the accompanying ulna fracture or radius

(c) The Author(s). 2019 Open Access This article is distributed under the terms of the Creative Commons Attribution 4.0 International License (http://creativecommons.org/licenses/by/4.0/), which permits unrestricted use, distribution, and reproduction in any medium, provided you give appropriate credit to the original author(s) and the source, provide a link to the Creative Commons license, and indicate if changes were made. The Creative Commons Public Domain Dedication waiver (http://creativecommons.org/publicdomain/zero/1.0/) applies to the data made available in this article, unless otherwise stated. 
head injury [5]. The associated extent of the capsuleligament injury can only be assumed. While during childhood the injury often heals with very good results, a complicative healing process is often observed for injuries in adults [6].

There is an agreement in the literature that the precise anatomical reconstruction of the ulna fracture is the key to a successful surgical therapy [7]. In general, the distal part of the ulna fracture that remains intact in the interosseous membrane leads to a reduction of the radius head in the elbow joint or in the PRUJ. In order to dislocate the radius head with intact capsule ligament structures of the humeroulnar joint part, the ligament connections between radius and ulna (consisting of annular ligament, chorda obliqua and proximal part of the interosseous membrane) must rupture at the level of the ulna fracture (Fig. 1). The distal part of the interosseous membrane of the fracture usually remains intact. Even after anatomical reduction and fixation of the ulna fracture, a persistent instability auf the PRUJ can remain (Fig. 2).

It is not known whether the torn ligament connections between the radius and ulna actually heal to a stable condition without surgical revision and under the obligatory early functional treatment. A standard surgical refixation of the torn annular ligament is not recommended [2, 8]. However, it can be assumed that with ulnar osteosynthesis alone, an instability of the radius head will remain in the PRUJ and in relation to the humeral capitulum. It can be assumed that their extent increases significantly from proximal to distal depending on the level of the ulna fracture.

The aim of the present work is to evaluate the extent of instability of the radius head in the PRUJ as a function of the severity of elbow fracture and ligament injury in an experimental and biomechanical approach.

\section{Methods \\ Specimens}

Eight fresh-frozen cadaver arms were provided by the Institute of Anatomy of the university clinic, Technical University Dresden, Germany. The specimens were frozen at $-22^{\circ} \mathrm{C}$ (Liebherr Typ 40073 1, Germany). Within the preparation, all soft tissues were removed except for the ligament structures of the PRUJ and forearm. The distal ulna was solid clamped in a vise. To reduce stability variation, the preparation was carried out by a single senior orthopaedic surgeon in a standardized fashion. Todisco and Trisi had already proven that Hounsfield units (HU) measured in CT correlate highly with bone mineral density [9]. Therefore, the bone density of the specimens was measured by using a quantitative computed tomography (Somatom CT, Siemens, München, Germany, technical specifications: CTDI 4.53 vol mGy, $\mathrm{kV}$ 80, mAs 180, $0.75 \mathrm{~mm}$ layer thickness). The bone density of all used proximal ulnas were in average $596 \pm$ 127 (min 495, $\max 891$ ) HU.

\section{Test setup and intervention}

A $4.5-\mathrm{mm}$ Schanz screw was inserted in a vertical direction and perpendicular in the horizontal plane. Clockwise markings at 3, 6 and 9 o'clock were applied to the radius head. Furthermore, the lowest point in the PRUJ was marked as a reference (Fig. 2). By means of a mechanical force measuring device (PGH, Kraftmessgeraete, Halle [Saale], Germany), a tensile force of $40 \mathrm{~N}$ was

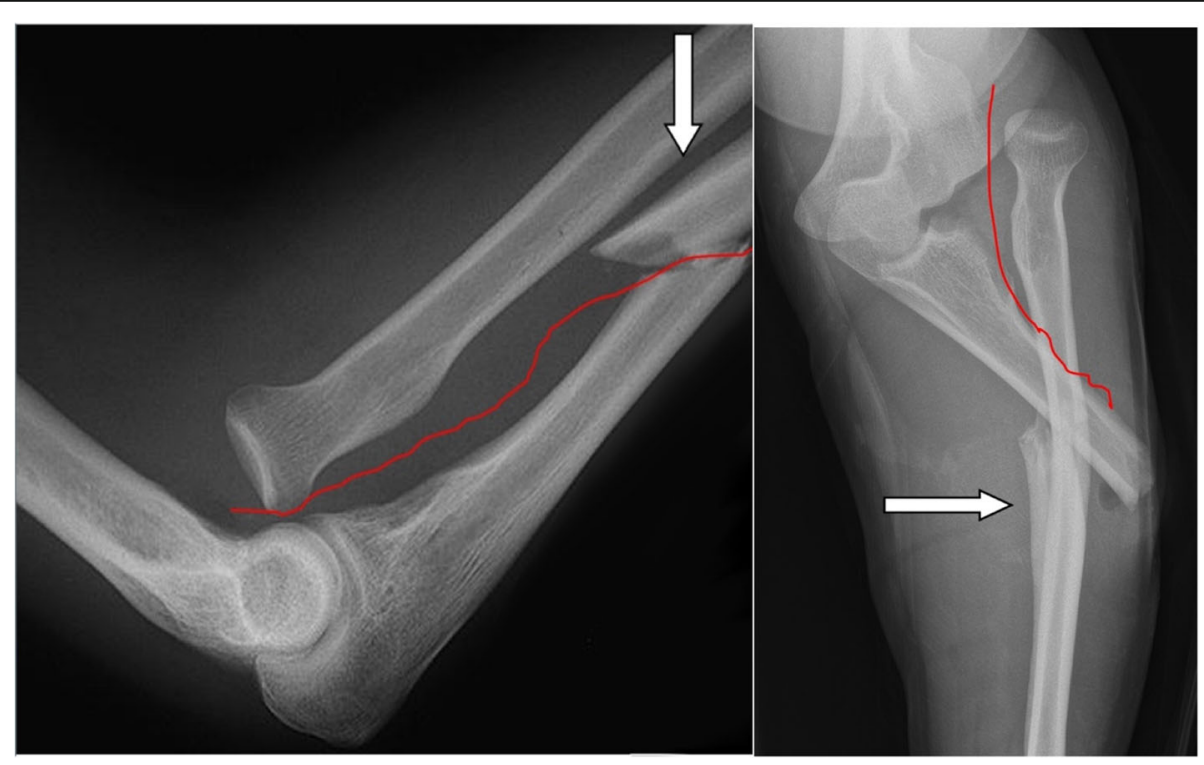

Fig. 1 X-ray of a Monteggia fracture (Bado I) with potential injury of the proximal ligamentary structures between the ulna and the radius including the PRUJ until the end of the distal ulnar fracture (white arrow) in left: lateral and right: anteroposterior view 


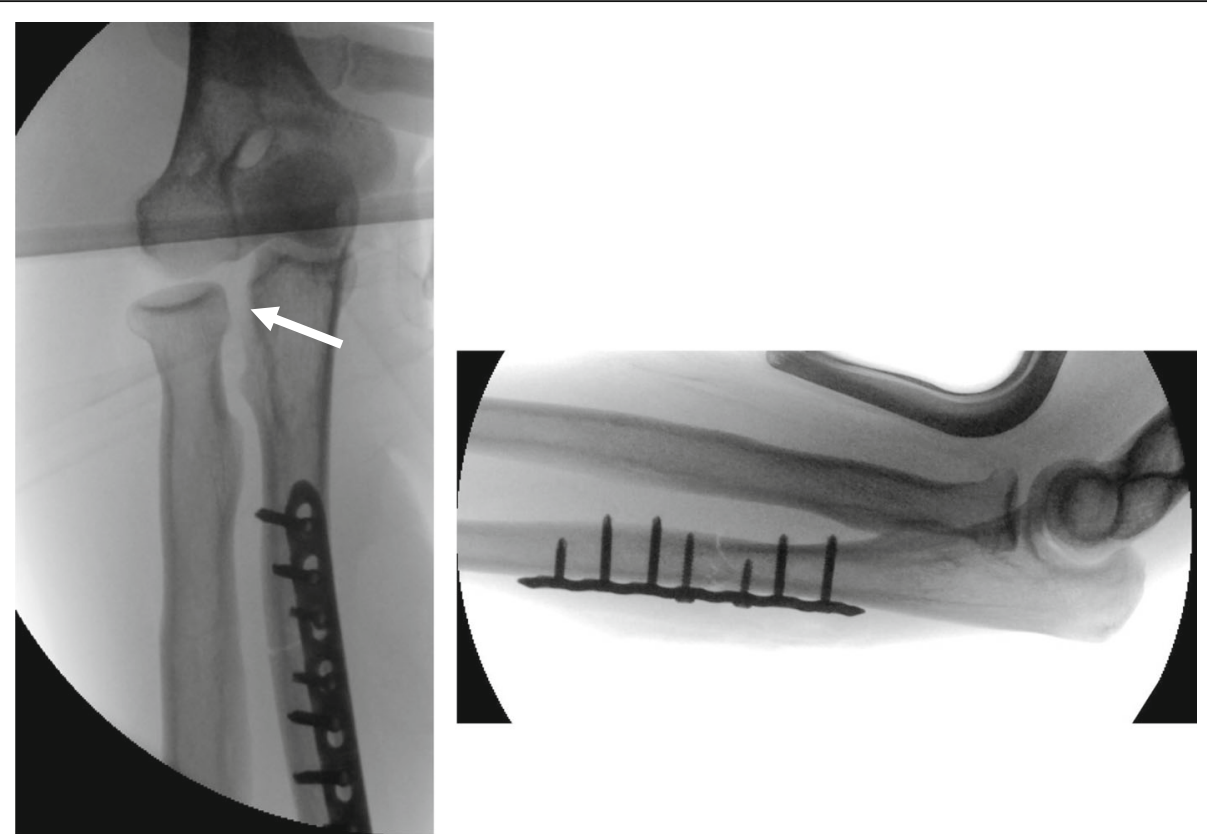

Fig. 2 Intraoperative X-ray after plate osteosynthesis of a Monteggia fracture with remaining instability in the PRUJ (white arrow)

exerted laterally, anteriorly or posteriorly on the proximal radius. The dislocation in the PRUJ was photometrically recorded and measured by two independent investigators using image processing software (Paint.net, $\operatorname{dotPDN}$ LLC).

After manual dissection of the ligament structures up to the interosseous membrane, the instability was documented and subsequently measured. The following dissection levels were differentiated (Fig. 3):
A. Intact ligament structures
B. Dissection of annular ligament
C. Additional dissection of the annular ligament and oblique cord

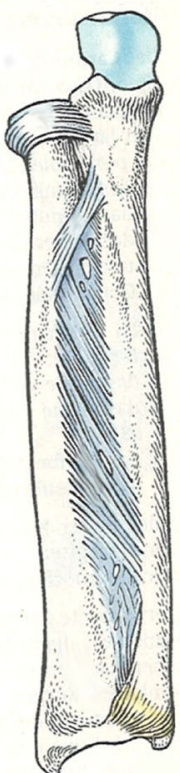

A

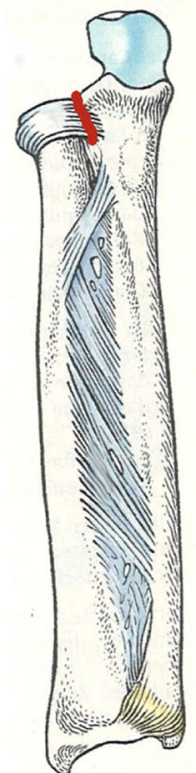

B

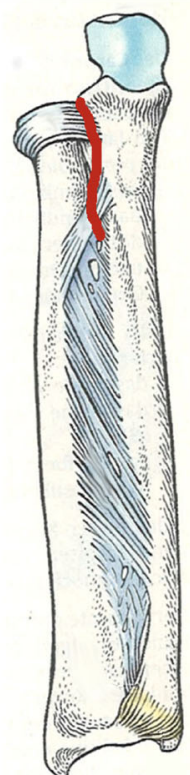

C

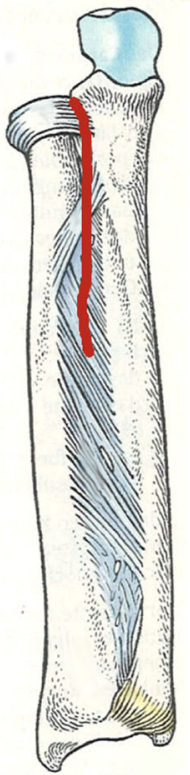

D

Fig. 3 Drawing of the forearm with the level of the dissection (red line). a Intact ligament structures. b Annular ligament. c Annular ligament and oblique cord. $\mathbf{d}$ Annular ligament, oblique cord and proximal third of interosseous membrane 
D. Additional dissection of annular ligament, oblique cord and proximal third of interosseous membrane

Statistical analysis was performed with SPSS Statistics software (version 25; IBM, Armonk, NY, USA) for descriptive statistics. The significance level was chosen at $p<0.05$. All data are presented as mean with standard deviation, minimum and maximum. Univariate analysis of variance was carried out to compare the different instabilities.

\section{Results}

The average age of the used donors was $81.6 \pm 9.4$ (6292) years. Five donors were female and three male. All biomechanical tests were successfully completed without the Schanz screws or the holding device loosening itself.

\section{Setting A (intact ligament structures)}

With intact ligament structures there is almost no instability in the PRUJ. It measures $1.5 \mathrm{~mm}$ (SD 1.08, min $0, \max 2.7$ ) in the anterior direction, $0.7 \mathrm{~mm}$ (SD 1.28, min 0, max 3.0) in the lateral direction and $1.6 \mathrm{~mm}$ (SD 1.57, $\min 0, \max 3.9)$ in the posterior direction. There was no significant difference among these groups.

\section{Setting B (dissection of annular ligament)}

After dissection of the annular ligament, instability occurs mostly posteriorly and slightly laterally. An anterior instability is almost not measured. An instability of 1.8 $\mathrm{mm}$ (SD 1.7, $\min 0, \max 4.2$ ) in the anterior direction, $4.1 \mathrm{~mm}$ (SD 2.7, $\min 2.4$, $\max 10.1$ ) in the lateral direction and $13.9 \mathrm{~mm}$ (SD 4.05, min 10.8, $\max 22.9$ ) in the posterior direction was recorded (Fig. 4).

\section{Setting C (dissection of the annular ligament and oblique cord)}

After dissection of the annular ligament and oblique cord, another posterior instability is generated. A lateral instability of $5.7 \mathrm{~mm}$ (SD 2.3, min 2.5, $\max 9.72$ ), an anterior instability of $2.9 \mathrm{~mm}(\mathrm{SD} 1.7, \min 0, \max 1.7)$ and a posterior instability of $17.5 \mathrm{~mm}$ (SD 6.3, min 10.5, $\max$ 26.5) were measured.

\section{Setting $D$ (dissection up to proximal third of interosseous membrane)}

After the dissection of the proximal third of the interosseous membrane, a massive multidirectional instability was observed in the lateral direction with dislocation of the radius head in the PRUJ in the posterior and anterior direction. In detail, there was a lateral instability of 10.3 $\mathrm{mm}$ (SD 2.6, $\min 6.7, \max 14.2$ ), an anterior instability of $15.8 \mathrm{~mm}$ (SD 5.3, $\min 9.2, \max 23.1$ ) and a posterior instability of $23.9 \mathrm{~mm}$ (SD 12, $\min 10.1, \max 45.2$ ).

\section{Instability in regard of direction}

Considering the instability in regard of the direction, it is noticeable that the anterior instability remains relatively constant until the proximal third of the interosseous membrane is dissected (Fig. 5). This is also seen statistically with a significant increase of the instability when dissecting the interosseous membrane $[p=.001]$. The early subluxation of the radius head in the posterior direction after dissection of the annular ligament with considerable stability to anterior and lateral is remarkable. In the course of our examinations, the posterior instability increased in inverse proportion with initial large increase of instability and decreases in regard of the distally resected structures (Fig. 7). However, the successive instability is always significant (Table 1). The lateral instability increases relatively constantly during the resection of the ligament structures distally. It is striking that a slight translation to the posterior direction always occurs with lateral traction. However, only the lateral offset was measured (Fig. 6).

\section{Discussion}

Precise ligamentous guidance of the radius rotating around the ulna is essential for free range of motion and painless strength of the forearm. The translation of the radius head during forearm rotation is therefore limited to only $1-2$ $\mathrm{mm}$ for intact ligaments between the ulna and the radius

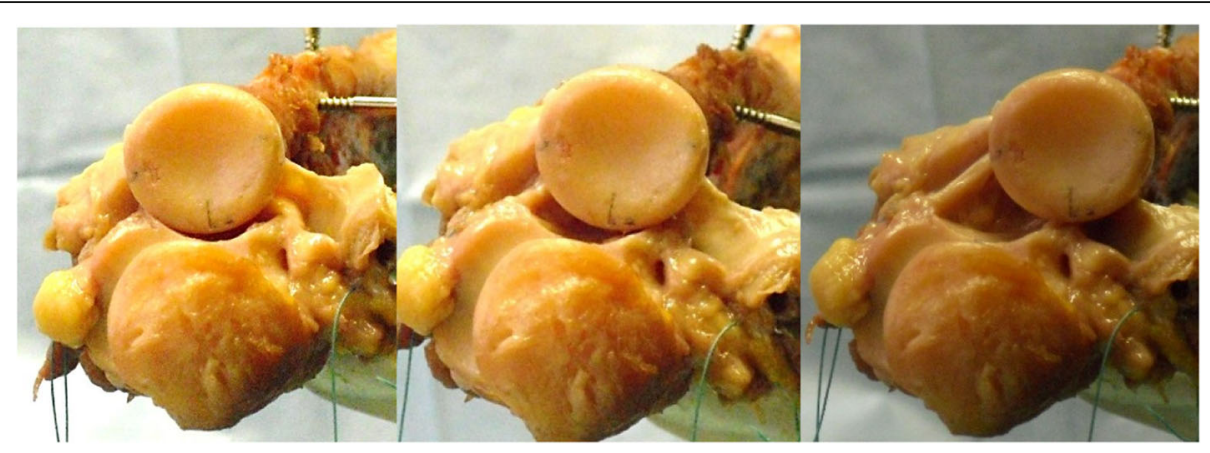

Fig. 4 Instability of the PRUJ dissection of the annular ligament (left: anterior, centre: lateral, right: posterior) 


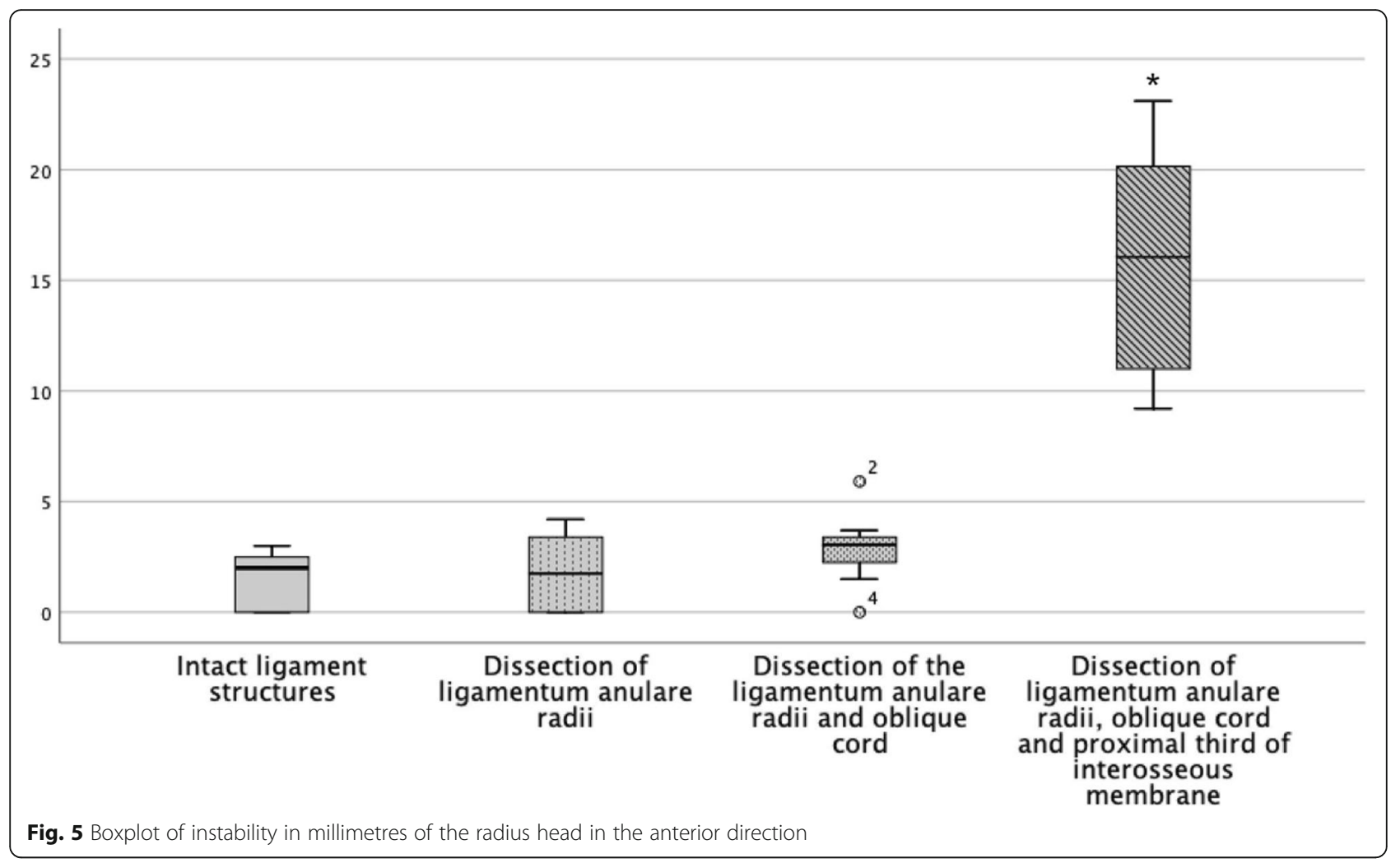

bone $[10,11]$. In case of Monteggia fractures, besides the anatomical reconstruction of the ulna fracture, the objective of the treatment must be the sufficient healing of the ligamentous structures in the PRUJ and the interosseous membrane.

In the literature, only three studies investigate experimentally the resulting instability in the PRUJ after cutting band structures [12-14]. All of these studies have evaluated the effect of ligamentary structure resection in regard of the stability in the PRUJ. In the study according to Galik et al., the translation of the radius head increased from $1.6 \pm 0.7$ to $2.3 \pm 0.9 \mathrm{~mm}$ in the mediolateral (ml) plane and from $2.1 \pm 0.6$ to $2.6 \pm 0.9 \mathrm{~mm}$ in the anteroposterior (ap) plane after severing the annular ligament during pro-/supination [12]. A direct comparison to the present study is difficult because only the sum of the distance in one plane (ap and $\mathrm{ml}$ ) was measured without the exact data for the anterior, lateral or posterior plane being given. In this study, however, the complete elbow joint in the $90^{\circ}$ position with intact lateral collateral ligament was tested, which also makes the comparability difficult, because the $90^{\circ}$ position of

Table 1 Overview of the measurement data

\begin{tabular}{|c|c|c|c|c|c|c|}
\hline Direction of dislocation & Involved structure & Instability in millimetres & Min & Max & SD & $p$ \\
\hline \multirow[t]{4}{*}{ Anterior } & Intact & 0.5 & 0 & 2.7 & 1.08 & \\
\hline & Annular ligament & 1.8 & 0 & 4.2 & 1.7 & .699 \\
\hline & Oblique cord & 2.9 & 0 & 1.7 & 1.7 & .219 \\
\hline & Interosseous membrane & 15.8 & 9.2 & 23.1 & 5.3 & .000 \\
\hline \multirow[t]{4}{*}{ Lateral } & Intact & 0.7 & 0 & 3.0 & 1.28 & \\
\hline & Annular ligament & 4,1 & 2.4 & 10.1 & 2.7 & .002 \\
\hline & Oblique cord & 5.7 & 2.5 & 9.72 & 2.3 & .031 \\
\hline & Interosseous membrane & 10.3 & 6.7 & 14.2 & 2.6 & .000 \\
\hline \multirow[t]{4}{*}{ Posterior } & Intact & 1.6 & 0 & 3.9 & 1.57 & \\
\hline & Annular ligament & 13.9 & 10.8 & 22.9 & 4.05 & .000 \\
\hline & Oblique cord & 17.5 & 10.5 & 26.5 & 6.3 & .047 \\
\hline & Interosseous membrane & 23.9 & 10.1 & 45.2 & 12 & .045 \\
\hline
\end{tabular}



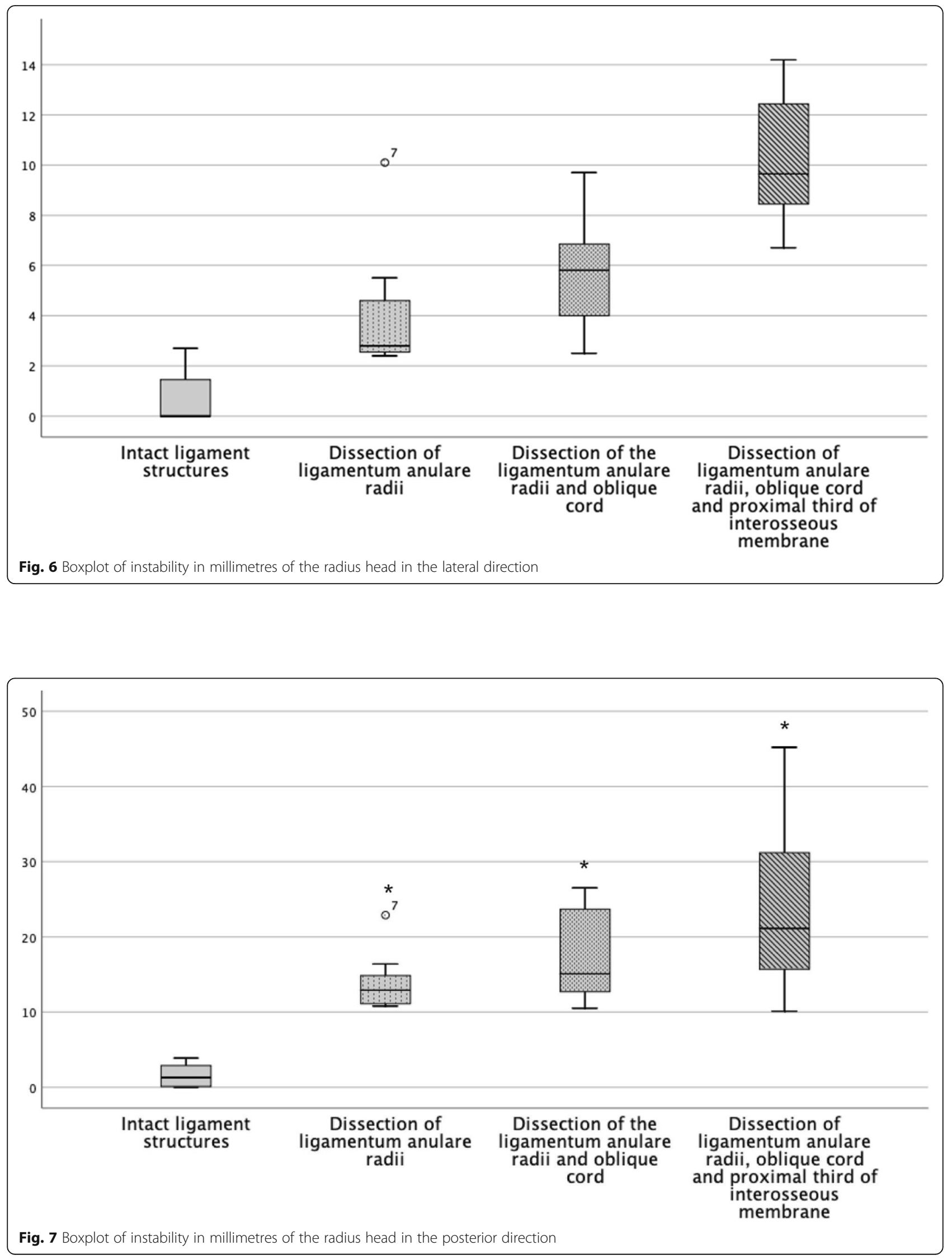
the elbow is a very stable position anyway when the primary stabilizing ligaments were not resected.

A comparable experimental setup has been chosen in the study of Anderson et al. The forearm including the elbow joint was examined and the ulnar collateral ligament, the lateral ulnar collateral ligament (LUCL) and the joint capsule of the elbow were left intact during preparation [13]. After dissection of the annular ligament, chorda obliqua and proximal interosseous membrane, the dislocation of the radius head in the PRUJ was measured in the lateral direction. Even after the dissection of all structures except for the distal interosseous membrane, the maximum diameter was only 3 (SD 2) $\mathrm{mm}$. Due to the intact primary ligamentary structures, the study is difficult to compare with the present study. However, there is no relevant instability in any direction in the PRUJ, which indicates in comparison to our study, that the not resected structures (ulnar collateral ligament, the LUCL and the joint capsule) contribute to considerable stability. In the present study, the instability of the PRUJ was therefore measured just by the use of forearm specimens without an annexed elbow joint and after resection of the medial und lateral ligament structures.

The resulting instability of the radius head was more obvious in the experimental approach of Galik et al. [12]. The elbow joint with capsule and ligament structures remained intact and the specimen was clamped in $90^{\circ}$ elbow flexion. The dislocation of the radius head in lateral, anterior and posterior plane after application of $20 \mathrm{~N}$ tensile force was measured and reported in percent of the diameter to the radius head. After dissection of the annular ligament, a significant lateral (46\%) and posterior (37\%) instability was measured, while stability in the anterior direction (8\%) was retained. The same results were seen in the present study with no significant instability in the anterior direction and already subluxation of the radius head in the lateral and posterior direction. However, in the study of Hayami et al., it was larger in the lateral direction, while in the present study, the largest instability was evaluated in the posterior direction after dissection of the annular ligament [14].

Not until the separation of the proximal half of the interosseous membrane, a subluxation was observed in the anterior direction (39\%) and even further in the lateral $(154 \%)$ and posterior $(200 \%)$ direction. In comparison to the present study, these results correspond precisely to the currently evaluated data. Also in the present study, a dislocation in the PRUJ in the lateral and posterior plane was evaluated significantly after resection up to the membrane interossea, whereas in the anterior direction, only a comparatively low dislocation was found. However, the results of these experimental studies can only be transferred to a very limited degree onto the instability of the PRUJ after Monteggia fractures. In particular, in the $90^{\circ}$ elbow flexion with intact collateral ligaments, the guidance of the concave radius head on the convexity of the humeral capitulum may result in a considerable secondary stability in the frontal and sagittal planes. The dislocation of the radius head often leads to significant ruptures of the elbow joint capsule and the radial collateral ligament complex, so that articular guidance of the radius head is not possible even after stable ulna osteosynthesis (Fig. 2).

The study has some limitations. On the one hand, in the present study, a different experimental setup was chosen (no $90^{\circ}$ position of the elbow) and the primary and secondary stabilizing structures such as the collateral ligaments and joint capsule with the distal humerus were resected. However, we believe that a stability bias is created by the very stable $90^{\circ}$ position of the elbow, especially since the relevant instabilities of the elbow are created starting at approximately $30^{\circ}$ extension. On the other hand, compared to Hayami et al., we measured with double the force $(20$ vs. $40 \mathrm{~N})$, so in the present study, the measured instability is higher compared to other studies [14]. Nevertheless, we believe that $40 \mathrm{~N}$ is more appropriate in relation to the forearm natural weight. A further limitation is the analogue, manual measurement of the instability by an image processing software, which can result in a latent inaccuracy. However, we have tried to reduce this by using two independent investigators. A measurement with an optical system would be preferable for future studies.

\section{Conclusion}

Based on our experimental observation and the study of Hayami et al., a complete healing of the instability of the radial head under functional treatment is hardly conceivable at least for ligamentous injuries up to the chorda obliqua or proximal interosseous membrane. A remaining instability of the proximal radius is a possible cause for the unsatisfactory clinical results after certain Monteggia fractures. Therefore, we recommend an intraoperative stress test of the PRUJ (equivalent to the syndesmosis stress testing) after anatomically stable osteosynthesis of the ulna, and, in case of persisting significant instability, an operative reconstruction of the annular ligament.

In addition, the present study may give a possible explanation (i.e. early dorsal radius head dislocation after dissection of annular ligament) why the Bado II injury is the most frequent type of Monteggia fractures.

\section{Abbreviations}

ap: Anteroposterior; CT: Computer tomography; Fig: Figure; HU: Hounsfield unit; LUCL: Lateral ulnar collateral ligament; PRUJ: Proximal radioulnar joint; SD: Standard deviation

Acknowledgements

None. 


\section{Authors' contributions}

$A B$ is the main author of the article. FB, TR and ET did the experimental evaluation and are responsible for the data collection. PK and KDS are the internal reviewers of the study. JN has done the primary statistical evaluation. All authors read and approved the final manuscript.

\section{Funding}

The study was not funded.

\section{Availability of data and materials}

The material and the data are made available.

\section{Ethics approval and consent to participate}

Approval for the study was granted by the local ethics committee (EK 51012019)

\section{Consent for publication}

Informed consent was obtained from all individual participants included in the study.

\section{Competing interests}

The authors declare that they have no competing interests.

\section{Author details}

'Orthopaedic-Traumatology Centre (OUC), Carl Gustav Carus University, Technical University Dresden, Fetscherstraße 74, 01307 Dresden, Germany. ${ }^{2}$ Institute of Anatomy, Carl Gustav Carus University, Technical University Dresden, Dresden, Germany. ${ }^{3}$ Orthopaedic-Surgery Centre (OCC), Tübingen, Germany. ${ }^{4}$ Centre for Translational Bone, Joint and Soft Tissue Research, Technical University Dresden, Dresden, Germany.

Received: 12 July 2019 Accepted: 6 September 2019

Published online: 28 November 2019

\section{References}

1. Monteggia GB. Istituzioni chirurgiche Milano; 1814.

2. Josten C, Freitag S. Monteggia and Monteggia-like-lesions: classification, indication, and techniques in operative treatment. Eur J Trauma Emerg Surg. 2009;35:296-304.

3. Ring D. Monteggia fractures. Orthop Clin North Am. 2013;44:59-66.

4. Bado JL. The Monteggia lesion. Clin Orthop Relat Res. 1967;50:71-86.

5. Jupiter JB, Leibovic SJ, Ribbans W, Wilk RM. The posterior Monteggia lesion. J Orthop Trauma. 1991;5:395-402.

6. Ring $D$, Jupiter JB, Waters PM. Monteggia fractures in children and adults. J Am Acad Orthop Surg. 1998;6:215-24.

7. Eathiraju S, Mudgal CS, Jupiter JB. Monteggia fracture-dislocations. Hand Clin. 2007;23:165-77 V.

8. Delpont $\mathrm{M}$, Louahem D, Cottalorda J. Monteggia injuries. Orthop Traumatol Surg Res. 2018;104:S113-S20.

9. Todisco M, Trisi P. Bone mineral density and bone histomorphometry are statistically related. Int J Oral Maxillofac Implants. 2005;20:898-904.

10. Baeyens JP, Van Glabbeek F, Goossens M, Gielen J, Van Roy P, Clarys JP. In vivo 3D arthrokinematics of the proximal and distal radioulnar joints during active pronation and supination. Clin Biomech (Bristol, Avon). 2006;21 Suppl 1:S9-12.

11. Kim HJ, Yi JH, Jung JW, Cho DW, van Riet R, Jeon IH. Influence of forearm rotation on proximal radioulnar joint congruency and translational motion using computed tomography and computer-aided design technologies. J Hand Surg Am. 2011:36:811-5.

12. Galik K, Baratz ME, Butler AL, Dougherty J, Cohen MS, Miller MC. The effect of the annular ligament on kinematics of the radial head. J Hand Surg Am. 2007:32:1218-24.

13. Anderson A, Werner FW, Tucci ER, Harley BJ. Role of the interosseous membrane and annular ligament in stabilizing the proximal radial head. J Shoulder Elb Surg. 2015;24:1926-33.

14. Hayami N, Omokawa S, lida A, et al. Biomechanical study of isolated radial head dislocation. BMC Musculoskelet Disord. 2017;18:470.

\section{Publisher's Note}

Springer Nature remains neutral with regard to jurisdictional claims in published maps and institutional affiliations.

Ready to submit your research? Choose BMC and benefit from:

- fast, convenient online submission

- thorough peer review by experienced researchers in your field

- rapid publication on acceptance

- support for research data, including large and complex data types

- gold Open Access which fosters wider collaboration and increased citations

- maximum visibility for your research: over $100 \mathrm{M}$ website views per year

At BMC, research is always in progress.

Learn more biomedcentral.com/submissions 